Special issue of the International Conference on Computational and Experimental Science and Engineering (ICCESEN 2014)

\title{
Photoneutron Dose Measurement in Radiotherapy Room
}

\author{
Ü. KArA ${ }^{a}$, A. Mesbahi ${ }^{b}$, I. AKkurT ${ }^{c *}$ \\ ${ }^{a}$ Suleyman Demirel University, Vocational School of Healt Services, Isparta, Turkey \\ ${ }^{b}$ Medical Physics Department, Medical School, Tabriz University of Medical Sciences, Iran \\ ${ }^{c}$ Suleyman Demirel University, Physics Department, Isparta, Turkey
}

\begin{abstract}
Cancer is one of the main disease to threat for human health. Besides surgery and medical treatment (chemotherapy), radiotherapy is commonly used treatment method to kill cancer cell. For this method high energy photon is required. Clinic Lineer Accelerator (cLINAC) relatively lower energy than other LINAC has been used to obtain photon via bremsstrahlung processes. When accelerated electron beam impinges to the thin target bremsstrahlung occurs and photon can be created. Those photons can be focused on cancer cell and kill cancer. In order to focus photon beam collimator materials in LINAC head generally contains heavy elements is used and the interaction of bremsstrahlung photon with the such heavy nuclei the neutron can be produced inside the treatment rooms. The threshold of the neutron production for those materals is about $10 \mathrm{MeV}$. As the neutron is more dangerous than photon it is important to determine neutron dose during radiotherapy treatment. In this study neutron dose rate has been measured and simulated in radiotherapy room.
\end{abstract}

DOI: 10.12693/APhysPolA.128.B-372

PACS: 87.56.- , 87.55.Qr

\section{Introduction}

Using bremsstrahlung photon is one of the main way to treat cancer cell. Bremsstrahlung photons are created in medical LINAC and the interaction of bremsstrahlung photon with the heavy nuclei that constitute the linac head shielding is the main process of neutron production inside the treatment rooms. The threshold of the neutron production for those materals is about $10 \mathrm{MeV}$. As the neutron is more dangerous than photon it is important to determine neutron dose during radiotherapy treatment. The neutron distribution depends on some parameters $[1,2]$ such as gantry angle. In this study neutron dose rate has been measured and simulated in radiotherapy room.
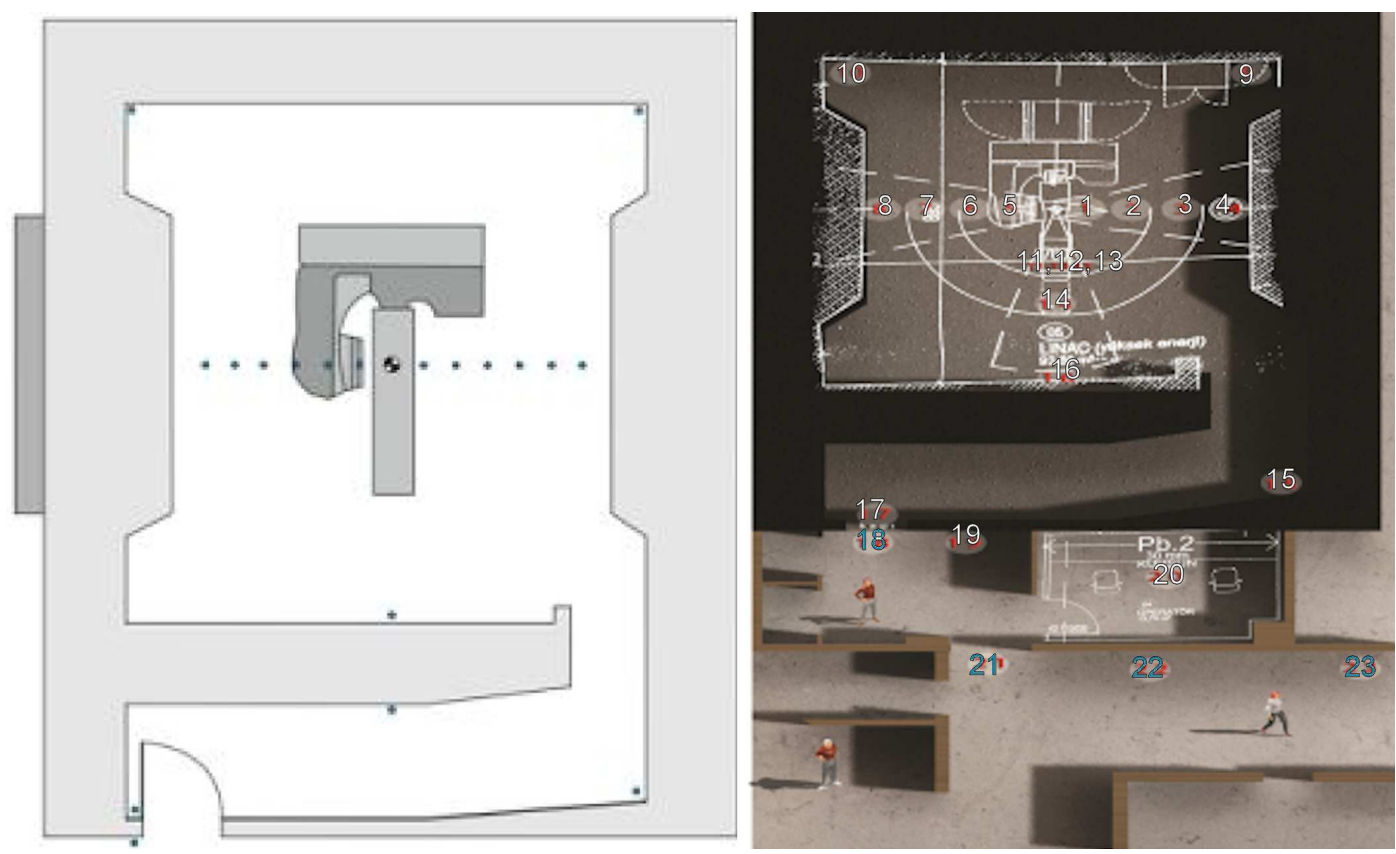

Fig. 1. Drawing and mock-up of medical linac examination point. 


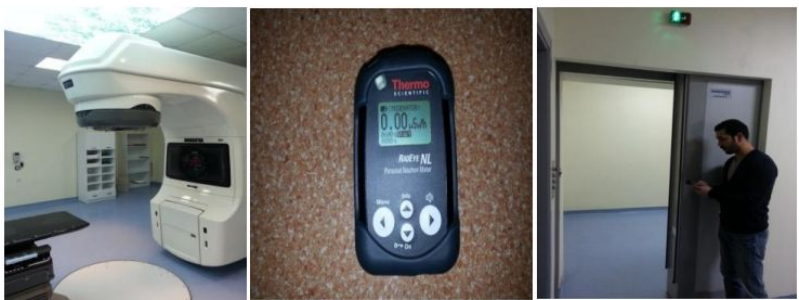

Fig. 2. Details of appliances used in measurements.
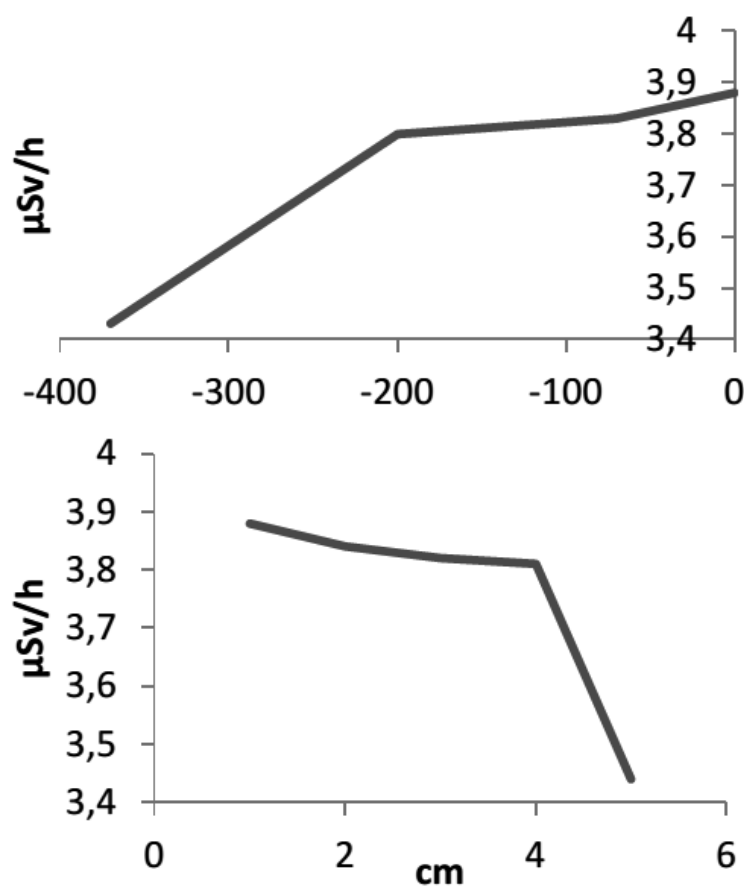

Fig. 3. Distance from central axis of beam $\left(0 \times 0 \mathrm{~cm}^{2}\right)$ : (top) points 1-4, (bottom) points 5-8.

\section{Material and method}

Radiation energy moves from one place to another. This is in the form of particles such as neutrons, while other times it is in the form of waves like $\mathrm{X}$ - or gamma rays. Radiations are grouped according to how much energy they contain and uses. Low energy radiation, like microwaves, is known as non-ionizing radiation. High energy radiation, such as X-rays, is known as ionizing radiation because it has enough energy to break chemical bonds and knock electrons (negatively charged particles) out of atoms. Photon is generated from the high-energy electron accelerators (LINAC) where an electron beam can be accelerated up to about $20 \mathrm{MeV}$ [3-9]. When electron beam impinged into thin metal photon is created via bremsstrahlung processes in medical hospital. Besides the known effects of radiation, the effects of surgical operations are still largely unknown. Thus it is
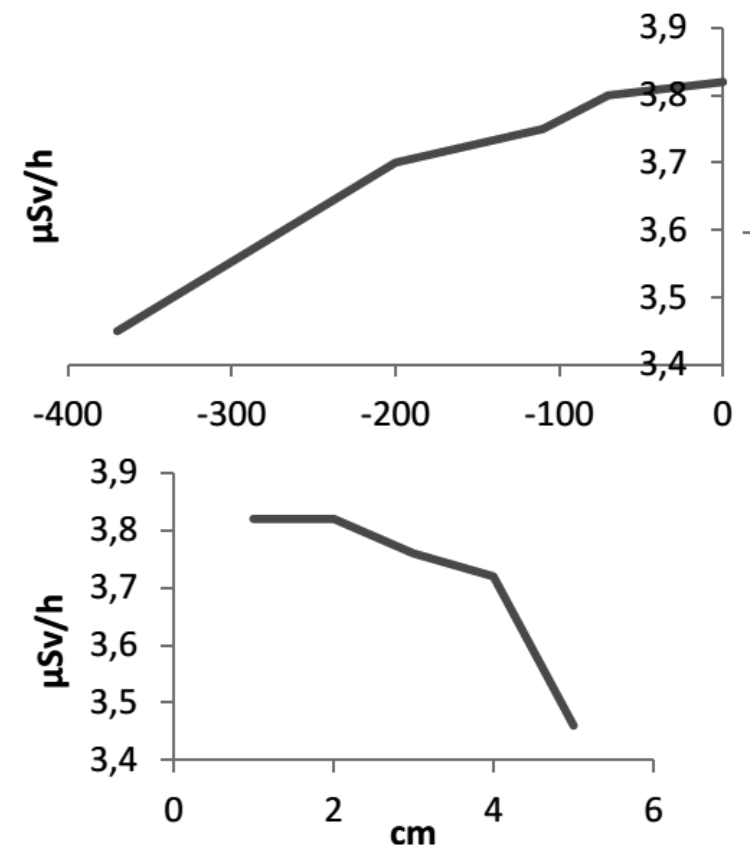

Fig. 4. Distance from central axis of beam $(20 \times$ $20 \mathrm{~cm}^{2}$ ): (top) points $1-4$, (bottom) points $5-8$.

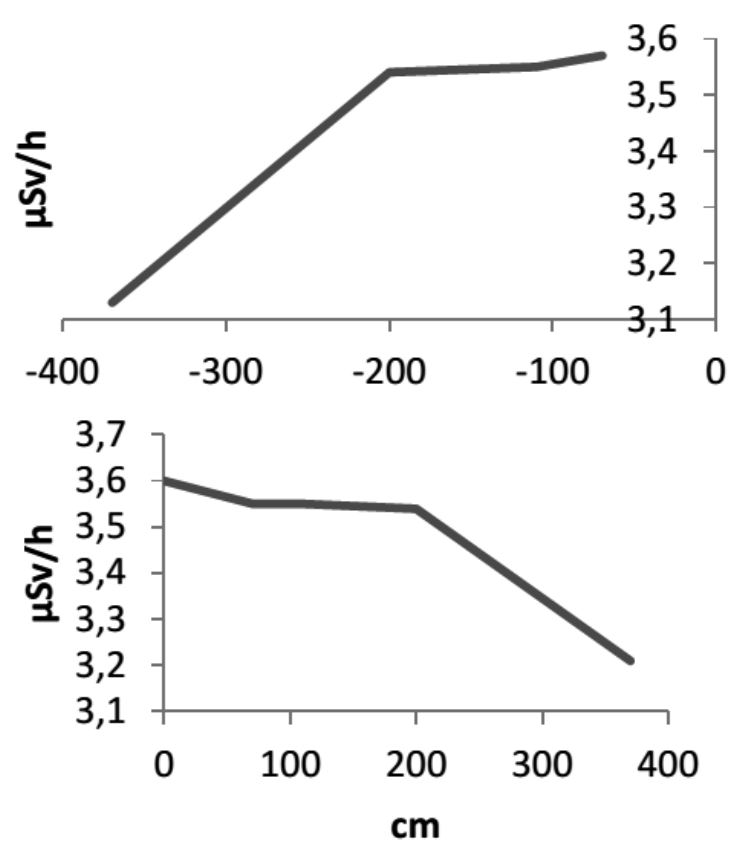

Fig. 5. Distance from central axis of beam $(40 \times$ $\left.40 \mathrm{~cm}^{2}\right)$ : (top) points $1-4$, (bottom) points $5-8$.

important and also difficult to measure doses of physicians and their patients during radioatherapy treatment. In this work, radiation dose due to the neutron contamination has been measured using neutron detector in radiotherapy room [5].

Radiation has existed since creation of universe and started to be used in a large of different fields nowadays. 


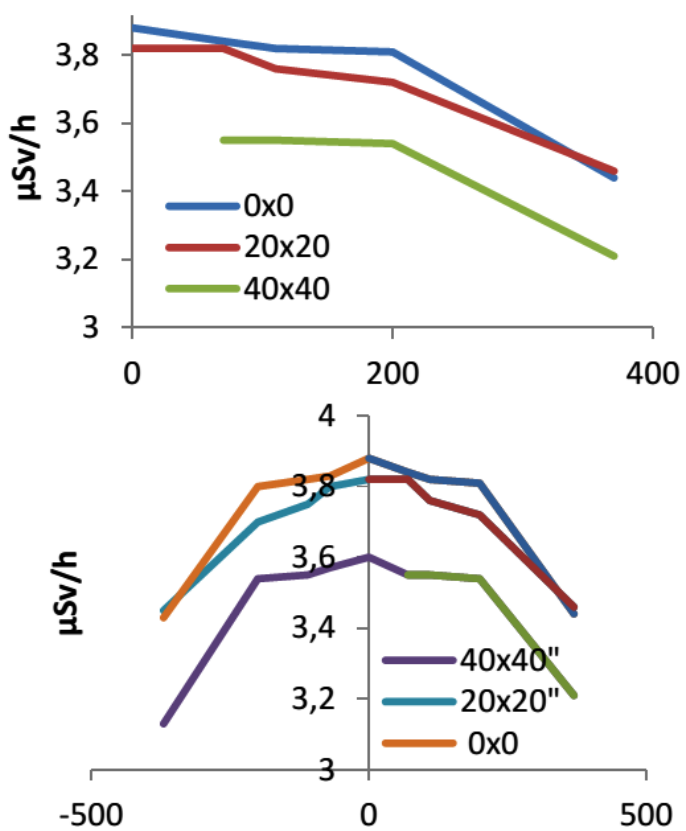

Fig. 6. The comparison with experimental measurements: (top) points 1-4, (bottom) points 1-8.

As human health is important one of the main used field of radiation is medical science for especially in the diagnostic and also treatment of diseases.

\section{Conclusion}

In this paper, we worked on Radiation Oncology Department in Suleyman Demirel Research Hospital. The threshold of the neutron production for those materals is about $10 \mathrm{MeV}$. As the neutron is more dangerous than photon it is important to determine neutron dose during radiotherapy treatment. The neutron distribution depends on some parameters such as gantry angle. In this study neutron dose rate has been measured as a function of gantry angle. Figures $3-6$ show neutron doses indexed with measuring points given at Fig. 1 .
In this study we measured Clinic Lineer Accelerator (cLINAC) the differences in cross sections used for bremsstrahlung effect on result in different photon spectra generated in the target and impinging on the surface of the phantom. However, the effect of these differences on dose computations are negligible.

\section{Acknowledgments}

This work has been supported partly by the Suleyman Demirel University Foundation Unit (3194-D212).

\section{References}

[1] The $200^{7}$ Recommendations of the International Commission on Radiological Protection, Ann. ICRP 37, 1 (2007).

[2] I. Akkurt, J.O. Adler, J.R. Annand, F. Fasolo, K. Hansen, L. Isaksson, Phys. Med. Biol. 48, 3345 (2003).

[3] A.J. Lenox, Radiat. Phys. Chem. 61, 223 (2001).

[4] Neutron contamination from medical accelerators, NCRP Report 79, 1984.

[5] A. Mesbahi, Appl. Radiat. Isot. 67, 55 (2009).

[6] European Guidance on Estimating Population Doses from Medical Ray Procedures, Radiation Protection 154, European Commission, Luxembourg 2008.

[7] L. Paredes, R. Genis, M. Balcázar, L. Tavera, E. Camacho, Radiation Measurements 31, 475-47 (1999).

[8] Fazal-ur-Rehman, H. Al-Ghamdi, M.I. Al-Jarallah, N. Maalej, Photoneutron Distributions around $18 \mathrm{MV}$ $X$-ray Radiotherapy Accelerators using Nuclear Track Detectors, King Fahd Unversity, Dhahran 2008.

[9] R.A. Geise, T.J. O'Dea, Appl. Radiat Isot. 50, 173 (1999). 